\title{
ARTHUR BYRON COBLE
}

\section{BY ARTHUR MATTUCK}

Professor Arthur Byron Coble, who was active in the American Mathematical Society from 1912 to 1940 , and its president in 19331934, died on December 8, 1966. Most of his professional work was done at the University of Illinois, and on April 1, 1968, the Urbana Senate recorded a memorial tribute to him which is reproduced here as an account of his life and interests. A description of his mathematical work has been added.

"Arthur Byron Coble was born in Williamstown, Pennsylvania in 1878. He completed his undergraduate studies at Gettysburg College in 1899 and received the Ph.D. degree from the Johns Hopkins University in 1902. During the academic year 1902-1903, he was an instructor at the University of Missouri, and he spent the following year in Germany as a Carnegie research assistant. He returned to Johns Hopkins in 1904 and remained there until 1918 when he was appointed Professor of Mathematics on this campus. Except for 1927-1928, which he spent at Johns Hopkins, he remained here until his retirement in 1947.

"Although he had little inclination for administrative responsibilities, he came to accept them as a necessary component of his work. From 1933 to 1947, he was head of the Department of Mathematics, and during these years he served on many University and College Committees, including eleven years on the University Council and eight years on the Executive Committee of the College of Liberal Arts and Sciences. He brought to these committees the calm judgment and the methodical analysis that characterized his work in other areas.

"Doing mathematics, however, was his prime pleasure. His first published paper appeared in 1901, and during the next four decades, in more than sixty articles and monographs, he developed his ideas and opened new areas of investigation. His work earned him distinction as a mathematician and pre-eminence in algebraic geometry, the area of his investigations. He received many honors from his professional colleagues. In 1928, he gave, by invitation, the Colloquium Lectures at the annual summer meeting of the American Mathematical Society, and his book Algebraic Geometry and Theta Functions, is Volume 10 of the Society's Colloquium Publications. In this book Professor Coble presents a very general and important 
connection between Cremona transformations and the theory of theta functions. His study of the geometric reality behind the formal properties of these functions, in the words of a reviewer, "is as varied as it is colorful" and "established connections as elegant as unexpected." He was elected to membership in the National Academy of Science; he served as editor or on the editorial board of the leading journals of mathematical research and as vice-president and president of the American Mathematical Society. He was awarded an honorary LLD degree by his alma mater.

"The years of Professor Coble's service to the University were years of growth in the Department of Mathematics. The number of Ph.D.'s awarded by the department increased from 10 during the 15 years preceding 1918 to 131 during the 30 years from 1918 to 1947 . Although he was heavily burdened with administrative duties, especially in the later years, he served as thesis advisor to 22 of the 131 successful candidates and as teacher to many more. His capacity for relating his work to many areas in mathematics, his incomparable talent and mathematical ingenuity, his dry humor and practical wisdom, all endeared him to his colleagues and his students.

"Arthur B. Coble was a man of diverse interests. He was a member of the University Club and participated regularly in its social programs. He was active in the American Association of University Professors and served on its investigating committees. His quiet selfeffacing personality is reflected in his reply to a professional colleague who requested biographical information. Coble wrote as follows: 'With respect to my hobbies, I can only say that they are essentially of a rather frivolous and time consuming character. I enjoy out-ofdoor sports-golf, tennis, swimming, and walking, and in bad weather I turn to bridge and billiards and light reading. I hope you can make something respectable out of this.'

"It is our pleasure to pay tribute to one who brought so much honor and distinction to the University.

Josephine Chanler

FRANZ E. HOHN

Clifford W. Mendel

HARRY LEVY"

\section{Mathematical Work}

Coble was primarily an algebraic geometer, and most of his work was involved in one way or another with the group $\mathrm{Cr}_{n}$ of Cremona

1 O. Zariski, Bull. Amer. Math. Soc. 36 (1930), 452-454. 
transformations. This is the group of birational transformations of projective $n$-space $P^{n}$ into itself; algebraically, it may be thought of as the group of automorphisms of a field $k\left(x_{1}, \cdots, x_{n}\right)$ of rational functions of $n$ variables.

The Cremona group is large and complicated. Consider for instance the case $n=2$. The group $\mathrm{Cr}_{2}$ of plane Cremona transformations contains the 8-dimensional subgroup consisting of the linear projective transformations, and according to a famous theorem it is generated as a group by these and one additional transformation, the classical quadratic transformation. Plane Cremona transformations are classified according to their "fundamental points" and their "type." The fundamental points are the finite set of points where the transformation is undefined. The classical quadratic transformation has three of these, for instance, and they always exist if the transformation is not linear. The "type" is a finite set of integers describing the behavior of the transformation at the fundamental points. In general the transformations with $n$ fundamental points and a given type depend on $2 n+8$ continuous parameters. Thus, $\mathrm{Cr}_{2}$ has both continuous and discrete features, and this makes it a fairly intractable group. The general Cremona group $\mathrm{Cr}_{n}$ is a good deal worse. Coble's review [4] of the enormous literature on Cremona transformations testifies to his encyclopedic grasp of the field, and at the same time to its scattered and unsatisfactory state.

One can look at the theory of $\mathrm{Cr}_{n}$ a sort of opposite to the theory of moduli. For in a general way, an algebraic variety either has moduli or it has automorphisms: either it has deformations into nearby varieties, or like $P^{n}$ it has deformations into itself. The current fashion is the study of moduli, and we know today little more about the Cremona group than Coble did 40 years ago.

One of Coble's chief preoccupations was the search for Cremona invariants, that is, properties of algebraic sets $V$ embedded in $P^{n}$ which are invariant under Cremona transformations of the ambient space. Of course if $V$ is irreducible, any birational invariant of $V$ will also be a Cremona invariant, but the problem is to find Cremona invariants which are not birational. Coble's answer was to study "point groups," that is, positive zero-cycles $P_{1}+\cdots+P_{k}$, the $P_{i}$ being points of the projective space. In a series of papers [20], [21], [25] he introduced an appropriate notion of equivalence ("congruence") for two such cycles under the Cremona group, and studied the resulting invariants. He succeeded in determining the finite number of types of Cremona transformations for which Cremona and projective equivalence coincide, and investigated various finite subgroups of $\mathrm{Cr}_{n}$ which arise in connection with certain zero-cycles. 
These cycles and subgroups of $\mathrm{Cr}_{n}$ run all through Coble's work in one guise or another. He was initially led to them by the use of invariant theory to solve the general 5 th and 6 th degree equations [9], [13], [14]. Here, the Galois group is represented as a group of Cremona transformations in the plane or space, whose fundamental points form part of a zero-cycle associated with the solutions of the original equation. In his major work, however, the zero-cycles are used to study the modular functions associated with algebraic curves of low genus. For example, a curve of genus 3 may be represented by a nonsingular plane quartic curve $C$. Counting multiplicities, there are 28 lines which are tangent to $C$ at 2 points. These lines correspond to 28 points in the dual projective plane, and from these one can canonically form a certain 288 zero-cycles of degree 7 . They are all Cremona equivalent, and the coordinates of their Chow points are modular functions of $C$.

These same zero-cycles also arose in the theory of theta modular functions, and Coble succeeded in establishing the connection between the two theories. His A.M.S. Colloquium volume, Algebraic Geometry and Theta Functions, exposes the theory for genus 2 and 3, and extends it to curves of genus 4 . These are represented as nonsingular curves $C$ of degree 6 in $P^{3}$, and one considers analogously the finite set of planes tangent to $C$ at three points. The book as a whole is a difficult mixture of algebra and analysis, as represented by classical invariant theory and theta functions, with intricate geometric reasoning of a type few can follow today. The calculations are formidable; let them serve to our present-day algebraic geometers, dwelling as they do in their Arcadias of abstraction, as a reminder of what awaits those who dare to ask specific questions about particular varieties.

In addition to the references cited, the symposium lecture [32] which enunciates Coble's viewpoint on Cremona transformations should be mentioned, as well as the more technical presidential address [50] on the Weddle surface.

\section{VITA}

CoBle, Arthur Byron (November 3, 1878-December 8, 1966)

Member of Council of A.M.S.

Member-at-large, 1912-1914

Vice-President, 1917

Member of Transactions Editorial Committee, 1920-1925

Chairman, Chicago Section, 1922-1923

President, 1933-1934 
Ex-President, 1935-1940

Member of Colloquium Editorial Committee, 1943-1944

Associate Editor of the Transactions, 1915-1919

Representative of the Society: On the Board of Editors of the American Journal of Mathematics, 1927-1933; On the American Section of the International Mathematical Union, 1920; Division of Physical Sciences, National Research Council, 1932-1935, 1946-1949.

Committees: Subcommittee on Invited Speakers, Committee on the Semicentennial Celebration, 1938; Committee on the Abstract Journal, 1938; Committee to Select Gibbs Lecturers for 1939, 1940; Committee to Select Hour Speakers for Annual and Summer Meetings, 1939; Nominating Committee, 1942; Committee on places of Meetings, 1941-1944; Committee to nominate officers and committees for the International Congress of Mathematicians, 1948.

Invited Addresses: Symposium on Cremona Transformations and Applications to Algebra, Geometry and Modular Functions, Chicago, April 1922; Cincinnati, December 1923; Amherst, September 1928 (Colloquium Lectures); New York, March 1934; Pittsburgh, December 1934 (Retiring Presidential Address); Durham, April 1939.

\section{BiBLIOGRAPHy}

1. On the reduction of the decimic to Sylvester's canonical form, Johns Hopkins University Circulars No. 3 (1901), 54-55.

2. The quartic curve as related to conics, Trans. Amer. Math. Soc. 4 (1903), 65-85.

3. Collineations whose characteristic determinants have linear elementary divisors with an application to quadratic forms, Amer. J. Math. 27 (1905), 25-46.

4. The normal form of a ternary collineation and the simultaneous reduction of two conics to a normal form, Johns Hopkins University Circulars No. 1 (1905), 27-28.

5. The linear relations among the minors of a symmetric determinant, Johns Hopkins University Circulars No. 9 (1906), 86-90.

6. On the relation between the three-parameter groups of a cubic space curve and a quadric surface, Trans. Amer. Math. Soc. 6 (1906), 1-20.

7. An invariant condition for certain automorphic algebraic forms, Amer. J. Math. 28 (1906), 333-366.

8. A configuration in finite geometry isomorphic with that of the 27 lines of a cubic surface, Johns Hopkins University Circulars No. 7 (1908), 80-88.

9. An application of the form-problems associated with certain Cremona groups to the solution of equations of higher degree, Trans. Amer. Math. Soc. 9 (1908), 183-212.

10. Symmetric binary forms and involutions, Amer. J. Math. 31 (1909), 183-212.

11. Symmetric binary forms and involutions. II, Amer. J. Math. 31 (1909), 355-364.

12. Symmetric binary forms and involutions. III, Amer. J. Math. 32 (1910), 333-364.

13. An application of Moore's cross-ratio group to the solution of the sextic equation, Trans. Amer. Math. Soc. 12 (1911), 311-325.

14. The reduction of the sextic equation to the Valentiner form-problem, Math. Ann. 70 (1911), 337-350. 
15. The lines and triple tangent planes of a cubic surface, Johns Hopkins University Circulars No. 2 (1911), 59-63.

16. The linear complex in the finite geometry (mod. 2) of an $S_{5}$, Johns Hopkins University Circulars No. 2 (1912), 43-46.

17. An application of finite geometry to the characteristic theory of the odd and even theta functions, Trans. Amer. Math. Soc. 14 (1913), 241-276.

18. Restricted systems of equations, Amer. J. Math. 36 (1914), 167-186.

19. Restricted systems of equations. II, Amer. J. Math. 36 (1914), 395-418.

20. Point sets and allied Cremona groups, Trans. Amer. Math. Soc. 16 (1915), 155198. Proc. Nat. Acad. Sci. U.S.A. 1 (1915), 245-248.

21. Point sets and allied Cremona groups. II, Trans. Amer. Math. Soc. 17 (1916), 345-385. Proc. Nat. Acad. Sci. U.S.A. 2 (1916), 244-246.

22. A proof of White's porism, Proc. Nat. Acad. Sci. U.S.A. 2 (1916), 530-531.

23. An isomorphism between theta characteristics and the (2p+2)-point, Ann. of Math. (2) 17 (1916), 101-112.

24. Point sets and allied Cremona groups, Trans. Amer. Math. Soc. 18 (1917), 331372. Proc. Nat. Acad. Sci. U.S.A. 2 (1918), 575-576. 340.

25. Theta modular groups determined by point sets, Amer. J. Math. 40 (1918), 317-

26. Concerning a method for finding a particular integral, Amer. Math. Monthly 26 (1919), 12-15.

27. The ten nodes of the rational sextic and the Cayley symmetroid, Amer. J. Math. 41 (1919), 243-265.

28. Multiple binary forms with the closure property, Amer. J. Math. 43 (1921), 1-19.

29. A covariant of three circles, Bull. Amer. Math. Soc. 27 (1921), 434-437.

30. Geometric aspects of the abelian modular functions of genus four. I, II, Proc. Nat. Acad. Sci. U.S.A. 7 (1921), 234-238, 245-249.

31. Cremona transformations and applications to algebra, geometry, and modular functions, Bull. Amer. Math. Soc. 28 (1922), 329-364.

32. Associated sets of points, Trans. Amer. Math. Soc. 24 (1922), 1-20.

33. Geometric aspects of the abelian modular functions of genus four. III, Proc. Nat. Acad. Sci. U.S.A. 9 (1923), 183-187.

34. Etude géomètrique des transformations birationelles et des courbes planes par Henri Malet, Bull. Amer. Math. Soc. 29 (1923), 38.

35. Geometric aspects of the abelian modular functions of genus four, Amer. J. Math. 46 (1924), 143-192.

36. The equation of the eighth degree, Bull. Amer. Math. Soc. 30 (1924), 301-313.

37. with H. R. Brahana, Maps of 12 countries with five sides with a group of order 120 containing an ikosahedral subgroup, Amer. J. Math. 48 (1926), 1-20.

38. Double binary forms with the closure property, Trans. Amer. Math. Soc. 28 (1926), 357-383.

39. with F. Morley, New results in elimination, Amer. J. Math. 49 (1927), 463488.

40. Topics in algebraic geometry. Chap. IV: Planar Cremona transformations; Chap. VIII: Cremona transformations in space and hyperspace, Bull. National Research Council No. 63 (1928), 79-121, 197-226.

41. Algebraic geometry and theta functions, Amer. Math. Soc. Colloq. Publ., vol. 10, Amer. Math. Soc., Providence, R. I., 1929; rev. ed., 1961. MR 23 \#A1279.

42. Geometric aspects of the abelian modular functions of genus four. II, Amer. J. Math. 51 (1929), 495-514.

43. A generalization of the Weddle surface, of its Cremona groups, and of its para- 
metric expression in terms of hyperelliptic theta functions, Amer. J. Math. 52 (1930), 439-500.

44. A treatise on algebraic plane curves, by J. L. Coolidge, Amer. Math. Monthly 29 (1932), 293-295.

45. Hyperelliptic functions and irrational binary invariants, Amer. J. Math. 54 (1932), 425-452.

46. Hyperelliptic functions and irrational binary invariants. II, Amer. J. Math. 55 (1933), 1-21.

47. Hyperelliptic functions and irrational binary invariants. III, Amer. J. Math. 55 (1933), 349-375.

48. Cremona's diophantine equations, Amer. J. Math. 56 (1934), 459-489.

49. The geometry of the Weddle manifold $W_{p}$, Bull. Amer. Math. Soc. 41 (1935), 209-222.

50. with Josephine Chanler, The geometry of the Weddle manifold $W_{p}$, Amer. J. Math. 57 (1935), 183-218.

51. Collineation groups in a finite space with a linear and a quadratic invariant, Amer. J. Math. 58 (1936), 15-34.

52. Groups of Cremona transformations in space of planar type, Duke Math. J. 2 (1936), 1-9.

53. Groups of Cremona transformations in space of planar type. II, Duke Math. J. 2 (1936), 205-219.

54. A class of linear groups with integral coefficients, Duke Math. J. 3 (1937), 175199.

55. Cremona transformations with an invariant rational sextic, Bull. Amer. Math. Soc. 45 (1939), 285-288.

56. Configurations defined by theta functions, Duke Math. J. 5 (1939), 479-488. MR 1, 27.

57. Trilinear forms, Duke Math. J. 7 (1940), 380-395. MR 3, 182.

58. Conditions on the nodes of a rational plane curve, Duke Math. J. 7 (1940), 396-410. MR 3, 183.

59. The double- $N_{n}$ configuration, Duke Math. J. 9 (1942), 436-449. MR 3, 305.

60. A particular set of ten points in space, Duke Math. J. 9 (1942), 450-453. MR 3, 305.

61. Ternary and quaternary elimination, Amer. J. Math. 68 (1946), 521-543. MR 8, 191.

62. On the expression of an algebraic form in terms of a set of forms with non-zero resultant, Amer. J. Math. 68 (1946), 544-552. MR 8, 191. 